This is an Open Access article distributed under the terms of the Creative Commons Attribution-Noncommercial License, which permits unrestricted use, distribution, and reproduction in any noncommercial medium, provided the original work is properly cited.

\title{
ENHANCED FAR-IR LINE EMISSION AT HIGH-Z: IMPLICATIONS FOR SPICA
}

\author{
R. Maiolino ${ }^{1}$, P. Caselli ${ }^{2}$, and G. De Zotti ${ }^{3}$ \\ ${ }^{1}$ INAF - Osservatorio Astronomico di Roma, via di Frascati 44, 00040 Monte Porzio Catone, Italy \\ ${ }^{2}$ University of Leeds, School of Physics and Astronomy, Leeds, LS2 9JT, UK \\ ${ }^{3}$ INAF - Osservatorio Astronomico di Padova, Vicolo dell'Osservatorio 5, 35122, Padova, Italy
}

\begin{abstract}
Recent observations of the fine structure line $[\mathrm{CII}] 158 \mu \mathrm{m}$ in high redshift galaxies have shown that this line is significantly stronger in distant galaxies than in local counterparts with the same far-IR luminosity. This enhancement at high-z probably applies also to other far-IR fine structure lines. This result suggests that SPICA, and in particular its spectrometer SAFARI, will be able to identify and characterize a much larger number of high-z galaxies through these far-IR lines than previously assumed. We discuss the new potentialities offered by SPICA for cosmological studies within this new scenario.
\end{abstract}

Key words: Galaxies: formation, evolution, starburst Missions: SPICA

\section{INTRODUCTION}

The far-IR spectral region $(50-200 \mu \mathrm{m})$ hosts the strongest lines in the spectrum of any galaxy. These are fine structure lines emitted by several species in various ionization stages (e.g. [CII] $158 \mu \mathrm{m},[\mathrm{OI}] 63 \mu \mathrm{m}$ and $145 \mu \mathrm{m}$, [NII] $122 \mu \mathrm{m}$, [OIII $] 88 \mu \mathrm{m}$ and $51 \mu \mathrm{m},[\mathrm{NIII}] 57 \mu \mathrm{m})$ and they are the main coolants of the interstellar medium (mostly in the photodissociation regions, PDRs, but also in the HII regions). As a consequence, their intensity is proportional to the heating rate, which is given by the UV radiation field of young stars, in star-forming galaxies. In particular, the cumulative intensity of the strongest far-IR lines is proportional to the star formation rate (Kaufman et al., 1999). The relative intensity of these lines also provides constraints on the metallicity of the ISM (Nagao et al., in prep.), on the density of the UV radiation field (Kaufman et al., 1999) and on the hardness of such field (i.e. AGN versus starburst excitation, Spinoglio et al., 2005). Since these are the strongest lines in the spectrum of any galaxy, they could in principle be used as a powerful tool to detect and identify the redshift of distant galaxies. However, although extremely strong, they cannot be observed from ground because in a spectral region where the atmosphere is opaque, while their detectability with current and past space observatories (e.g. Herschel, ISO) is mostly limited to local galaxies (except possibly for a few strongly lensed sources). The greatly enhanced capabilities of SPICA will open the possibility of using these far-IR lines for the detection, identification and characterization of high redshift galaxies. In the following we discuss the expected intensity of the far-IR lines in high-z galaxies and their detectability with SPICA, and with its spectrometer SAFARI in particular.

\section{ENHANCED FAR-IR LINE EMISSION AT HIGH-Z}

Among the far-IR fine structure lines, the $[\mathrm{CII}] 158 \mu \mathrm{m}$ line is generally the strongest and the best studied by past farIR space and airborne observatories. This single line generally accounts for as much as $0.1-1 \%$ of the total bolometric luminosity of galaxies (Crawford et al., 1985; Stacey et al., 1991; Wright et al., 1991). Although with a large spread, the [CII] luminosity is proportional to the far-IR luminosity of galaxies, with $-3 \leq \log \left(\mathrm{L}_{[\mathrm{CII}]} / \mathrm{L}_{\mathrm{FIR}}\right) \leq-2$, at least for $\mathrm{L}_{\mathrm{FIR}}<10^{11} \mathrm{~L}_{\odot}$ (Stacey et al., 1991). However, for sources with $\mathrm{L}_{\mathrm{FIR}}>10^{11}-10^{11.5} \mathrm{~L}_{\odot}$ the ratio $\log \left(\mathrm{L}_{[\mathrm{CII}]} / \mathrm{L}_{\mathrm{FIR}}\right)$ drops by an order of magnitude (Malhotra et al., 2001; Luhman et al., 1998, 2003; Negishi et al., 2001), as illustrated in Figure 1. This effect has been often interpreted as a reduced heating efficiency of the ISM in strong radiation fields (Kaufman et al., 1999), but other scenarios have also been proposed (see Maiolino et al., 2005, for a summary). Regardless of the physical origin of the effect, the sharp drop of the $\mathrm{L}_{[\mathrm{CII}]} / \mathrm{L}_{\mathrm{FIR}}$ ratio at high luminosities has cast doubts over the usefulness of the $[\mathrm{CII}]$ line to trace high-z galaxies.

Although locally the [CII] $158 \mu \mathrm{m}$ line can only be observed from space or from airborne observatories, at high redshift the line is shifted into the submm-mm windows of atmospheric transmission, and therefore can be observed with groundbased facilities. Recently, mm and submm observations have achieved the first few detections of [CII] at high redshift (Maiolino et al., 2005; Iono et al., 2006; Walter et al., 2009; Maiolino et al., 2009). These detections are currently achieved only in the host galaxies of quasars, with far-IR luminosities $\mathrm{L}_{\mathrm{IR}}>10^{12} \mathrm{~L}_{\odot}$ (i.e. in the range of Ultra Luminous Infrared Galaxies, ULIRGs). One of the most surprising results is that high-z galaxies appear to be characterized by enhanced [CII] emission relative to local galaxies. This is illustrated in Figure 1, where the location of high-z galaxies (squares) in the $\mathrm{L}_{[\mathrm{CII}]} / \mathrm{L}_{\mathrm{FIR}}$ versus $L_{F I R}$ diagram is well above the extrapolation of the steeply declining trend observed in local, luminous galax- 
ies (solid line). With the currently available data, at a given luminosity, the [CII] enhancement in high-z galaxies relative to local counterparts appears to be nearly an order of magnitude.

The origin of this effect is still not clear yet. A possibility is that it is a consequence of the lower metallicity of the ISM in high-z galaxies. Observationally, local lowmetallicity galaxies tend to show enhanced [CII] $158 \mu \mathrm{m}$ emission (Rubin et al., 2009; Poglitsch et al., 1995; Israel et al., 1996; Madden, 2000). The effect is apparent from the location of LMC in Figure 1 (Rubin et al., 2009), which shows an enhancement of [CII] by a factor of about four relative to more metal rich galaxies. Probably the enhanced [CII] emission in low metallicity galaxies stems from the lower dust content (hence lower dust attenuation to UV photons), which makes the C[II]- emitting region larger, and also makes the far-IR emission lower $(\mathrm{Ru}-$ bin et al., 2009). Regardless of the nature of the effect, if high-z galaxies are characterized by a reduced metallicity, this may enhance their [CII] emission similarly to local low-metallicity galaxies. High-z star-forming galaxies are indeed observed to have lower gas metallicities than local galaxies (Maiolino et al., 2008). For galaxies hosting quasars, such as those currently detected in [CII] at high-z, the situation is more complex. Various studies have found that the metallicity in the broad line region (BLR) of high-z quasars is very high (several times solar) and does not evolve with redshift (Juarez et al., 2009; Jiang et al., 2007; Nagao et al., 2006a). However, the BLR is a very tiny region $(<1 \mathrm{pc})$ in quasar nuclei, which is probably not representative of the ISM in the host galaxy, and may undergo quick enrichment with just a few supernova explosions (see detailed discussion in Juarez et al., 2009). A few studies have investigated the metallicity on the larger scales $(\sim 100 \mathrm{pc}-10 \mathrm{kpc})$ of the narrow line region (NLR) in high-z AGNs (Nagao et al., 2006b; Matsuoka et al., 2009; Vernet et al., 2001; De Breuck et al., 2000; Humphrey et al., 2008). Although these studies are currently limited to $\mathrm{z}<4$, the inferred NLR metallicities are much lower than in the BLR, about solar or sub-solar, which is indeed less than the metallicity observed in local massive galaxies (Maiolino et al., 2008).

Regardless of the physical origin of the [CII] enhancement in high-z galaxies, if confirmed this effect would have important implications for the planning of future surveys at high-z, as well as for the development of future far-IR and submm $/ \mathrm{mm}$ facilities. This result would imply that, at least at high infrared luminosities, the [CII] line in high$\mathrm{z}$ galaxies is a factor of several stronger than previously expected based on local templates. The [CII] line is probably a much more powerful cosmological tool to detect and characterize high-z galaxies than previously thought.

The behavior of the other far-IR fine structure lines is still unknown at high redshift. If the enhanced emission of [CII] is due to the low metallicities at high-z, then a similar enhancement is also expected for most of the other far-
IR lines. There are several observing programs (many of which involving our team) that are attempting to detect other far-IR lines in high-z galaxies. Preliminary results are encouraging and suggest that most far-IR lines are stronger at high-z relative to local counterparts (at a given far-IR luminosity). This has important consequences for the SPICA surveys at high-z, and in particular surveys with the SAFARI spectrometer, since it implies that many of the far-IR lines accessible to SPICA at high-z are likely much stronger than previously expected.

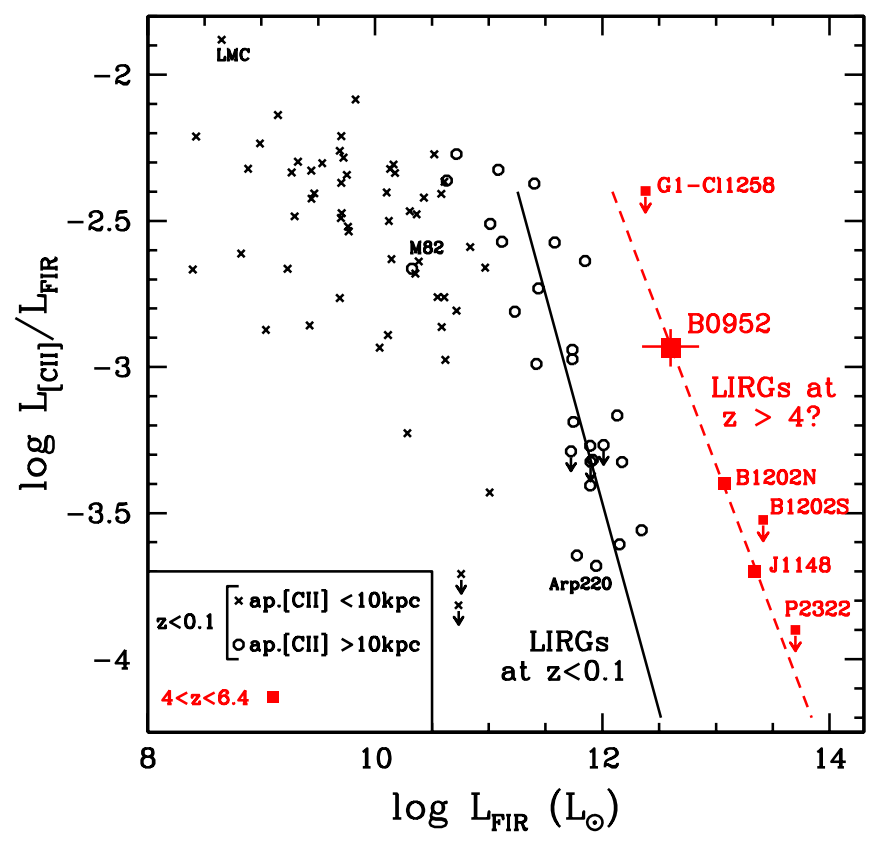

Figure 1. $\mathrm{L}_{[\mathrm{CII}]} / \mathrm{L}_{\mathrm{FIR}}$ ratio versus $\mathrm{L}_{\mathrm{FIR}}$ for normal and starburst local galaxies (crosses and circles) and for high-z sources (squares), from Maiolino et al. (2008). The black solid line shows a linear fit (of the logarithmic quantities) to the data of local luminous Infrared galaxies (LIRGs, $\mathrm{L}_{\mathrm{FIR}}>10^{11} \mathrm{~L} \odot$ ). The red dashed line is a linear fit to the detections at high redshift.

\section{IMPLICATIONS FOR SPICA AND SAFARI}

To estimate the number of sources for which SPICA is expected to detect far-IR lines, some assumptions must be made on the behavior of these lines at low luminosities in high-z galaxies, a range not yet probed by current observations. In the following we assume the "high-z enhanced scenario", where high-z sources with $\mathrm{L}_{\mathrm{FIR}}<10^{12} \mathrm{~L}_{\odot}$ are expected to have [CII] emission enhanced relative to local galaxies by a factor of about four, i.e. similar to the enhancement observed in the LMC (Figure 1), while at high luminosities $\left(\mathrm{L}_{\mathrm{FIR}}>10^{12} \mathrm{~L}_{\odot}\right)$ the $[\mathrm{CII}]$ enhancement matches current observations (Figure 1). Then, the luminosity of other far-IR lines are scaled to the [CII] lu- 
minosity based on ratios observed in local galaxies (e.g. Spinoglio et al., 2005; Luhman et al., 2003; Unger et al. 2000; Colbert et al., 1999; Metcalfe et al., 2005; Fischer et al., 1996; Negishi et al., 2001).

Under these assumptions, SAFARI is expected to detect far-IR lines, such as [OI] $63 \mu \mathrm{m}$ and [OIII $] 52 \mu \mathrm{m}$, in galaxies with $\mathrm{L}_{\mathrm{FIR}}=10^{11} \mathrm{~L}_{\odot}$ out to $\mathrm{z} \sim 2$ (i.e. around the peak of cosmic star formation) in one hour of integration. The same lines are detectable in lower luminosity $(\sim$ Milky Way-like) galaxies with $\mathrm{L}_{\mathrm{FIR}}=10^{10} \mathrm{~L}_{\odot}$ out to $\mathrm{z} \sim 1$ in a few hours of integration.

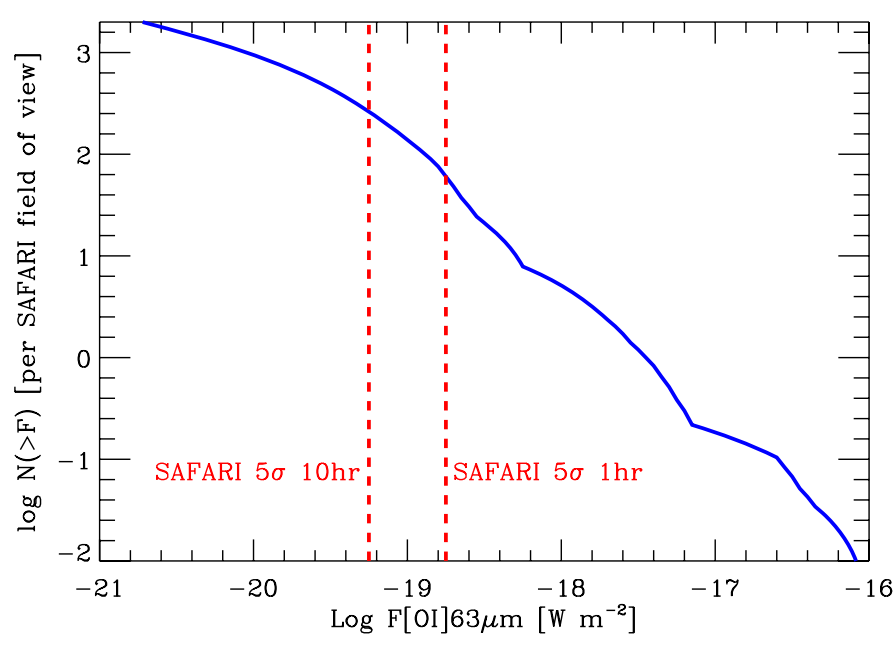

Figure 2. Integral source counts (per SAFARI field of view) for

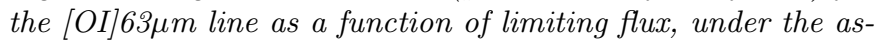
sumption that the [OI] line luminosity is enhanced at high-z as the [CII] line (see text).

To infer the number of galaxies expected to be detected in these lines, we used the cosmic evolution of the far-IR luminosity obtained by the models in Lapi et al. (2006) and Silva et al. (2005), which match current observational constraints at high redshift. Then, the evolution of the far-IR lines luminosity was determined through the line-to-FIR luminosity ratio inferred in the "enhanced scenario" discussed above. In the following we focus on the [OI $] 63 \mu \mathrm{m}$ line, which is generally the strongest far-IR line after [CII], and which is observable with SAFARI out to $\mathrm{z} \sim 2.3$. Figure 2 shows the expected integral source counts (per SAFARI field of view) as a function of the limiting flux for the $[\mathrm{OI}] 63 \mu \mathrm{m}$ line. The dashed vertical lines show the expected SAFARI sensitivity around the wavelength of $[\mathrm{OI}]$ at $\mathrm{z} \sim 1-2$, in 1 and 10 hours of integration, showing that SAFARI, with typical deep exposures, will be able to detect of the order of about a hundred sources per pointing. This implies that a typical deep survey of "only" a few hundred hours is expected to detect over ten thousand galaxies (most of which at $\mathrm{z} \sim 1-2$ ) in $[\mathrm{OI}] 63 \mu \mathrm{m}$ and other far-IR lines with similar intensity. With such a massive redshift survey, SPICA will be able to derive the three-dimensional clustering of galaxies around the peak of cosmic star formation, by also including heavily obscured galaxies (missed by optical and near-IR surveys), with important implications for the study of the evolution of the cosmic structures. The far-IR lines detected with SAFARI in these large sample of sources will allow us to characterize these distant galaxies in terms of star formation rate, metallicity and excitation mechanism (starburst versus AGN). SAFARI will therefore allow studies similar to those currently performed or planned by some extensive optical redshift surveys, but at higher redshift and without biases against dust-obscured sources.

\section{ACKNOWLEDGEMENTS}

Part of this work was supported by the Italian Institute for Astrophysics (INAF) and by the Italian Space Agency (ASI) through contract ASI-INAF I/016/07/0.

\section{REFERENCES}

Colbert, J. W., et al. 1999, ApJ, 511, 721

Crawford, M. K., Genzel, R., Townes, C. H., \& Watson, D. M. 1985, ApJ, 291, 755

Curran, S. J. 2009, A\&A, 497, 351

De Breuck, C., Röttgering, H., Miley, G., van Breugel, W., \& Best, P. 2000, A\&A, 362, 519

Fischer, J., et al. 1996, A\&A, 315, L97

Humphrey, A., Villar-Martín, M., Vernet, J., Fosbury, R., di Serego Alighieri, S., \& Binette, L. 2008, MNRAS, 383,11

Iono, D., et al. 2006, ApJ, 645, L97

Israel, F. P., Maloney, P. R., Geis, N., Herrmann, F., Madden, S. C., Poglitsch, A., \& Stacey, G. J. 1996, ApJ, 465, 738

Jiang, L., Fan, X., Vestergaard, M., Kurk, J. D., Walter, F., Kelly, B. C., \& Strauss, M. A. 2007, AJ, 134, 1150

Juarez, Y., Maiolino, R., Mujica, R., Pedani, M., Marinoni, S., Nagao, T., Marconi, A., \& Oliva, E. 2009, A\&A, 494, L25

Kaufman, M. J., Wolfire, M. G., Hollenbach, D. J., \& Luhman, M. L. 1999, ApJ, 527, 795

Lapi, A., Shankar, F., Mao, J., Granato, G. L., Silva, L., De Zotti, G., \& Danese, L. 2006, ApJ, 650, 42

Luhman, M. L., et al. 1998, ApJ, 504, L11

Luhman, M. L., et al. 2003, ApJ, 594, 758

Madden, S. C. 2000, New Astronomy Review, 44, 249

Maiolino, R., et al. 2005, A\&A, 440, L51

Maiolino, R., et al. 2008, A\&A, 488, 463

Maiolino, R., Caselli, P., Nagao, T., Walmsley, M., De Breuck, C., \& Meneghetti, M. 2009, A\&A, 500, L1

Malhotra, S., et al. 2001, ApJ, 561, 766

Matsuoka, K., Nagao, T., Maiolino, R., Marconi, A., \& Taniguchi, Y. 2009, A\&A, 503, 721

Metcalfe, L., et al. 2005, A\&A, 444, 777

Nagao, T., Marconi, A., \& Maiolino, R. 2006a, A\&A, 447, 157 
Nagao, T., Maiolino, R., \& Marconi, A. 2006b, A\&A, 447, 863

Negishi, T., Onaka, T., Chan, K.-W., \& Roellig, T. L. 2001, A\&A, 375, 566

Poglitsch, A., et al. 1995, ApJ, 454, 293

Rubin, D., et al. 2009, A\&A, 494, 647

Silva, L., De Zotti, G., Granato, G. L., Maiolino, R., \& Danese, L. 2005, MNRAS, 357, 1295

Spinoglio, L., Malkan, M. A., Smith, H. A., GonzálezAlfonso, E., \& Fischer, J. 2005, ApJ, 623, 123

Stacey, G. J., Geis, N., Genzel, R., Lugten, J. B., Poglitsch, A., Sternberg, A., \& Townes, C. H. 1991, ApJ, 373, 423

Unger, S. J., et al. 2000, A\&A, 355, 885

Vernet, J., et al. 2001, A\&A, 366, 7

Walter, F., Riechers, D., Cox, P., Neri, R., Carilli, C., Bertoldi, F., Weiss, A., \& Maiolino, R. 2009, Nature, 457, 699

Wright, E. L., et al. 1991, ApJ, 381, 200 